\title{
Refractory Ascites after Liver Transplantation: A Stepwise Approach to Diagnosis and Treatment
}

\author{
Kulkarni RD ${ }^{1}$, Thomas EM ${ }^{2}$, Zendejas $\mathbf{I}^{3}$, Fair $\mathrm{JH}^{1}$ and Andreoni KA ${ }^{2 *}$ \\ ${ }^{1}$ Department of Surgery, University of Texas Medical Branch, USA \\ ${ }^{2}$ Department of Surgery, University of Florida, USA \\ ${ }^{3} I M C$, Canyon Surgical Associates, USA
}

Submission: November 21, 2016; Published: December 06, 2016

*Corresponding author: Kenneth A Andreoni, Department of Surgery, University of Florida, 1600 SW Archer Rd, Gainesville, FL 32607, Tel: 352-265-0606; Fax: 352-265-0678; Email: kandreoni@ufl.edu

\begin{abstract}
Persistent ascites four weeks after successful liver transplant defines refractory ascites, an uncommon but a challenging problem. Etiology of the ascites can occur in the presence of cirrhotic or non-cirrhotic grafts. The first step in evaluation of refractory ascites after liver transplant includes a simultaneous evaluation of the characteristics of ascites, echocardiogram and duplex ultrasound of the graft vasculature. In portal hypertensive ascites both portal vein (PV) and or hepatic veins (HV) /vena cava abnormalities must be evaluated as the etiology. Short of massive biliary necrosis and bile peritonitis the hepatic artery plays little role in refractory ascites. Abnormalities on the duplex ultrasound usually lead the clinician to the most appropriate diagnostic and therapeutic intervention. We offer a simple algorithm based on the differentiation between cirrhotic and non-cirrhotic liver grafts. In non-cirrhotic liver grafts, we then expand upon the diagnosis and management of the most common etiology of refractory ascites, venous abnormalities.
\end{abstract}

Keywords: Ascites, Liver transplant, Portal hypertension, Venous stenosis, Venous obstruction

Abbreviations: PV: Portal Vein; RHV: Right Hepatic Vein; MHV: Middle Hepatic Vein; LHV: Left Hepatic Vein; RA: Refractory Ascites; OLT: Orthotopic Liver Transplant; HCV: Hepatitis C; HBV: Hepatitis B; CT: Computed Tomography; PHT: Portal Hypertension; PVT: Portal Vein Thrombosis; SMV: Superior Mesenteric Vein; TPA: Tissue Plasminogen Activator; TIPS: Transjugular Intrahepatic Porto-systemic Shunt; POD: Post- Operative Day; LFT: Liver Function Test

\section{Introduction}

Ascites is a common complication of advanced liver disease [1], and refractory ascites (RA) occurs when it cannot be satisfactorily treated by medical therapy. RA is known to carry a poor prognosis, with a 1 year transplant-free survival in advanced cirrhotic patients of 20-50\% [2]. Patients with RA usually have additional complications, including spontaneous bacterial peritonitis, hepatorenal syndrome and hepatic hydrothorax $[3,4]$.

RA after liver transplantation is uncommon and requires a thoughtful approach to diagnosis and treatment. Refractory ascites is defined according to the International Ascites Club [1] as persistent ascites present for more than 4 weeks after successful orthotopic liver transplant (OLT) and is seen in $3.4 \%$ to $5.9 \%$ of patients $[5,6]$. RA post-transplant may be due to parenchymal disease, carcinomatosis or a host of hemodynamic abnormalities [5,7].

\section{Mechanism of ascites in cirrhotic liver grafts}

The pathophysiology of ascites as a result of liver graft cirrhosis is the same as that in native liver cirrhosis. Early graft dysfunction from non-anatomical causes include recurrent hepatitis C (HCV; strongest association with a $3 \mathrm{x}$ increase in risk), hepatitis $\mathrm{B}(\mathrm{HBV})$, or decreased liver vascular compliance during acute / chronic cellular rejection $[7,8]$.

The accumulation of ascitic fluid represents a state of totalbody sodium and water excess, but the event that initiates the imbalance is unclear. Three theories of ascites formation have been proposed: underfilling, overflow, and peripheral arterial vasodilation. The underfilling theory suggests that the primary 
abnormality is inappropriate sequestration of fluid within the splanchnic vascular bed due to portal hypertension (PHT) and a consequent decrease in effective circulating blood volume. This activates the plasma renin-aldosterone and sympathetic nervous system, resulting in renal sodium and water retention.

The overflow theory suggests that the primary abnormality is inappropriate renal retention of sodium and water in the absence of volume depletion. This theory was developed in accordance with the observation that patients with cirrhosis have intravascular hypervolemia rather than hypovolemia.

The most recent theory, the peripheral arterial vasodilation hypothesis, includes components of both of the prior theories. It suggests that portal hypertension leads to vasodilation, which causes decreased effective arterial blood volume. As the natural history of the disease progresses, neurohumoral excitation increases, more renal sodium is retained, and plasma volume expands. This leads to overflow of fluid into the peritoneal cavity. The vasodilation theory proposes that underfilling is operative early and overflow is operative late in the natural history of cirrhosis [9-12].

Although the sequence of events that occurs between the development of portal hypertension and renal sodium retention is not entirely clear, portal hypertension apparently leads to an increase in nitric oxide levels [13]. Nitric oxide mediates splanchnic and peripheral vasodilation. Hepatic artery nitric oxide synthase activity is greater in patients with ascites than in those without ascites.

Regardless of the initiating event, a number of factors contribute to the accumulation of fluid in the abdominal cavity. Elevated levels of epinephrine and norepinephrine are welldocumented factors. Hypoalbuminemia and reduced plasma oncotic pressure favor the extravasation of fluid from the plasma to the peritoneal fluid, and, thus, ascites is infrequent in patients with cirrhosis unless both portal hypertension and hypoalbuminemia are present.

\section{Mechanism of ascites in non-cirrhotic liver grafts}

The etiology of post liver transplant ascites can be multifactorial. The most common anatomic issues are inadequate venous outflow from the liver graft, such as stenosis of the hepatic veins and/or vena cava anastomoses or inflow abnormalities such as portal vein stenosis or thrombosis (PVT) $[5-7,14,15]$. The use of reduced grafts or 'small for size' grafts maycause inadequate accommodation of liver blood flow and relative portal hypertension. Other causes include functional liver outflow obstruction from right heart failure, severe malnutrition and underlying renal failure $[7,16]$.

The literature contains several single center series with descriptions of the cause of refractory ascites post liver transplant; however, none gives a step wise approach to the diagnosis and management of this challenging problem.

\section{Illustrative Case Report}

A 54 y/o female with hepatitis C cirrhosis and hepatocellular carcinoma underwent orthotopic liver transplantation without peri-operative complications. Her transplant was performed in a piggyback fashion with supra-hepatic donor vena cava anastomosed to all three recipient hepatic veins. The liver graft was profoundly right lobe dominant. Her immediate post-operative ultrasound revealed patent vessels and her post-operative course was unremarkable for the first week. However, on post-operative day (POD) 7 her total bilirubin was increasing and a repeat ultrasound showed a small perihepatic fluid collection and a possible right hepatic vein thrombus. A contrasted CT scan was obtained which confirmed the thrombus (Figure 1). A heparin drip was started to prevent thrombus propagation and a venogram was performed revealing right hepatic vein obstruction at the site of the anastomosis. Attempts to traverse the occlusion by transjugular and transhepatic approaches were unsuccessful. She was transitioned to warfarin and discharged home with normal liver function tests (LFTs).

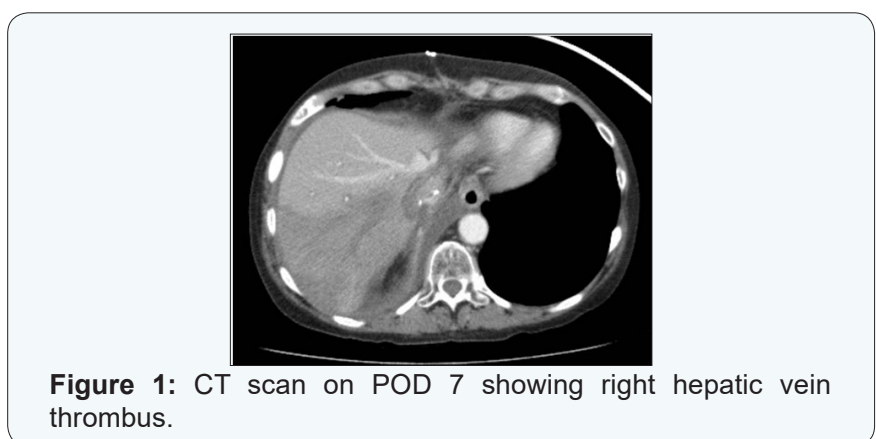
thrombus.

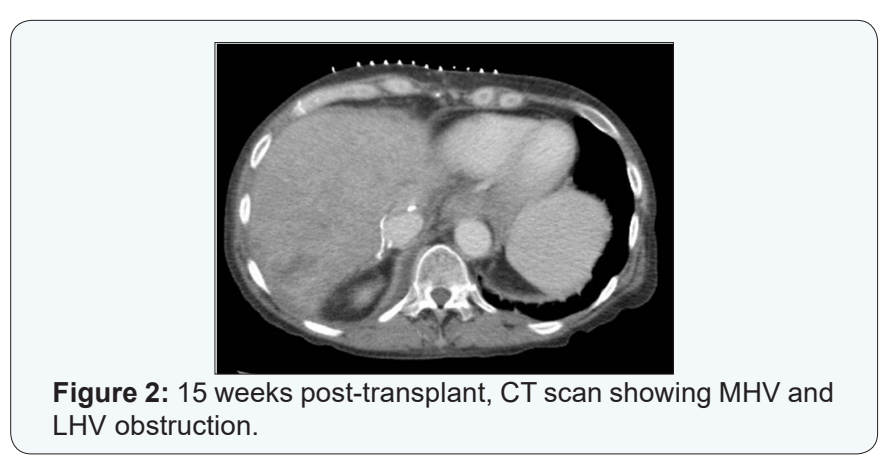

Six weeks post-transplant, a transjugular venogram with balloon angioplasty of the left, middle and right hepatic veins (LHV, MHV, RHV) was performed. The RHV/ right atrial (RA) gradient was reduced from $7 \mathrm{mmHg}$ to $4 \mathrm{mmHg}$ and the MHV / RA gradient was reduced from 5-6 mmHg to 1-2 mmHg. A month later, another venogram was performed demonstrating complete occlusion of the RHV. Angioplasty of the MHV was successful with a resultant gradient of $0 \mathrm{mmHg}$. Despite these treatments, she developed newly symptomatic ascites over the following 2 months. A CT scan obtained revealed moderate ascites and stable RHV thrombosis with patent MHV and LHV. At 15 weeks posttransplant, she presented with abdominal pain and distention with a repeat CT scan revealing RHV occlusion and high grade 
LHV and MHV stenosis due to mass effect from the congested right lobe (Figure 2). She was admitted for serial paracentesis and medical management of her worsening ascites.

After failure of medical management of her ascites, it was determined that the patient might benefit from a mesocaval shunt to decompress the portal hypertension given stable liver graft function. Because there was some risk to liver function with this procedure, the patient was relisted for liver transplant with a low MELD score prior to attempting the surgical shunt. To construct the mesocaval shunt, a $14 \mathrm{~mm}$ Goretex ${ }^{\circledR}$ graft was used as a conduit between the superior mesenteric vein (SMV) and infra-renal vena cava (Figure $3 \mathrm{~A}$ ). The patient tolerated the procedure well and the patient was discharged home two weeks after the procedure with stable graft function. CT scan done two weeks later revealed resolution of the ascites and widely patent shunt (Figure 3B).

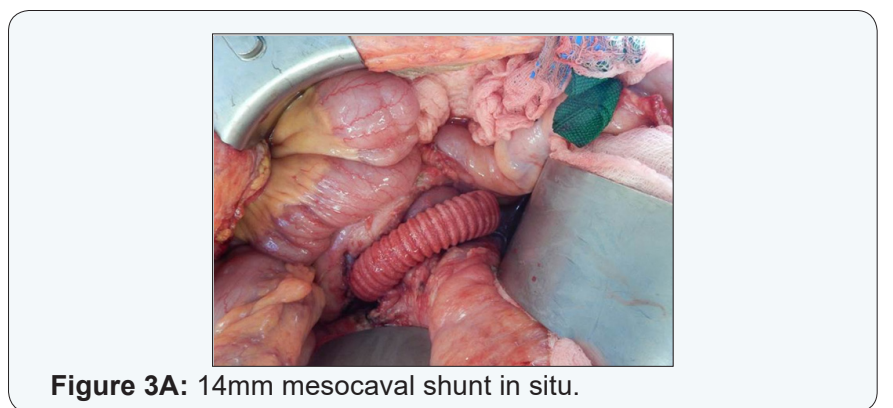

(4mm mesocaval shunt in situ.

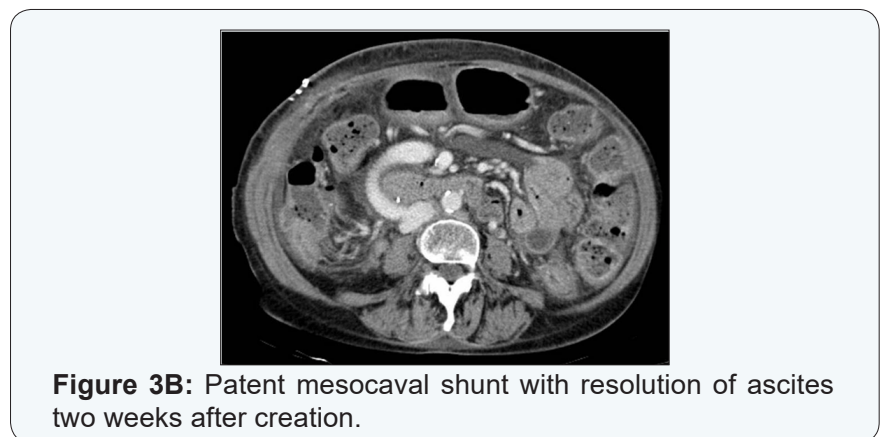

\section{Discussion}

Orthotopic liver transplantation is considered the best therapeutic option for end-stage liver disease, with excellent survival and substantial improvement in quality of life. The postoperative period is characterized by a high incidence of medical and surgical complications, including graft rejection, bacterial infections, and vascular or biliary problems, all of which have been extensively described. However, the development of ascites after transplantation has received less attention.

RA post liver transplant can have physiologic or anatomic origin. In our practice, we evaluate both of these potential etiologies in parallel. For physiologic causes, an echocardiogram evaluates heart function. Paracentesis is performed with gram stain, cell count/cytology, and cultures to evaluate for infection. Also, the serum-ascites albumin gradient (SAAG) is calculated to differentiate between portal hypertension (PHT) vs nonPHT causes of RA, including malignancy. In patients with a past history of HCC with elevated AFP levels, AFP can be obtained. A hypercoagulable work up should be entertained. A liver biopsy is performed with suspicion of parenchymal disease which may include graft rejection, recurrence of viral or autoimmune hepatitis, or cirrhosis.

To evaluate the anatomic causes, a duplex ultrasound (DUS) of the liver is performed which is highly sensitive and specific for determining vascular insult $[17,18]$. The DUS demonstrates whether there is an inflow (PV) or an out flow (HV) abnormality, based on wave forms (monophasic through triphasic appearance), absolute velocities, and step-up velocities across an anastomosis. A tri-phasic CT scan can be considered to better delineate the anatomy. However, if the DUS is of good quality a venogram can be performed directly avoiding a CT scan with its contrast load.

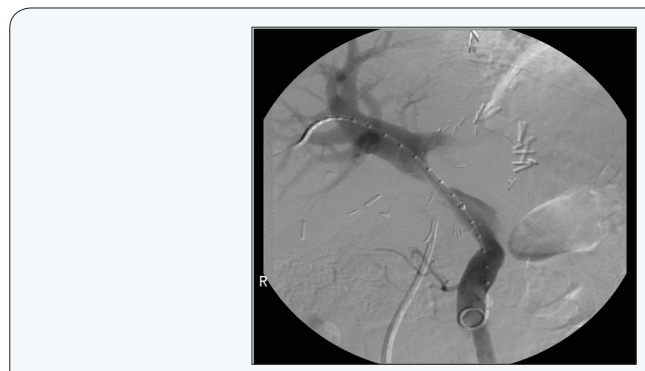

Figure 4A: Anastomotic PV stricture with catheter tip in superior mesenteric veinto measure pressure gradient.

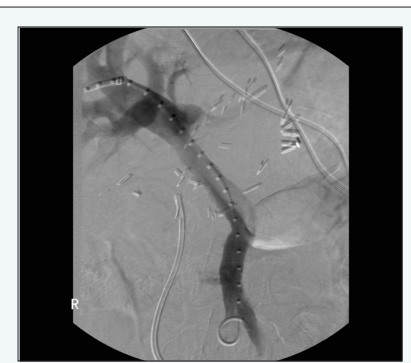

Figure 4B: Successful stent deployment in PV to relieve pressure gradient.

If the DUS shows an inflow abnormality as suggested by low velocity flows $(<20 \mathrm{~cm} / \mathrm{sec})$ or a step up velocity $>4$ across an anastomosis, a transhepatic portogram is performed. Portography will define the portal anatomy in terms of its patency and presence of porto-systemic shunts, while also allowing for direct portal pressure and gradient measurements with concurrent venoplasty if necessary. Problems with the portal vein could involve thrombosis, anastomotic stricture or kinking due to excessive length. With limited thrombosis of the portal vein, interventional radiology can attempt mechanical thrombolysis followed by tissue plasminogen activator (TPA), and if necessary, coiling of large portosystemic shunts if PV flow is inadequate due to steal. If intrahepatic portal veins are involved or attempted thrombolysis of the main portal vein is unsuccessful, then surgical intervention can be considered (thrombectomy / 
retransplant). Anastomotic strictures with $>5 \mathrm{~mm} \mathrm{Hg}$ gradient can be balloon venoplastied (Figure 4A). Regular follow up with duplex US is required due to reported stricture recurrence as high as $28.6 \%$ to $36.8 \%$ following a single balloon angioplasty alone. PV stricture may require repeat balloon venoplasties or eventual stent placement [19-21].Portal Vein stents do carry a significant thrombosis rate, so PV stent placement should only be done after several attempts at venoplasty alone and close discussion with the transplant surgical team (Figure 4B).

Obstruction due to kinking of vein can be treated with stents, but again, PV stenting has a high rate of thrombosis and stenting will also make surgical repair of the anastomosis more challenging. DUS and/or portograms are required for follow up after all significant venous interventions.

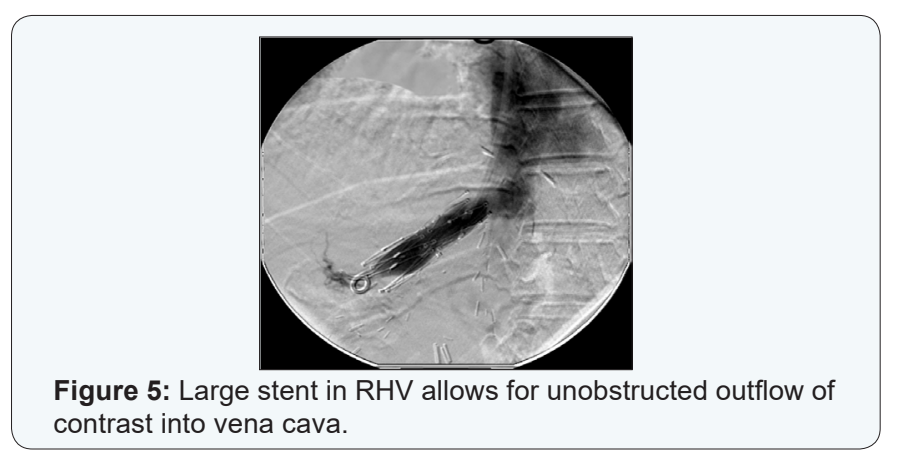

In case of an outflow abnormality on DUS, a transjugular hepatic venogram is performed. The venogram allows one to obtain direct imaging and pressure gradients. The transjugular approach allows for pressure measurement at the right atrium, suprahepatic and infrahepatic cava, hepatic veins, and finally a wedged hepatic vein as an indirect estimate of the PV pressure. A liver biopsy can also be performed in the same setting which can identify rejection or recurrent disease, especially in HCV. Thrombosis of one or more hepatic veins can be treated with mechanical thrombolysis and stent placement (Figure 5). However the success rate is limited. For anastomotic strictures, balloon venoplasty may be attempted with or without stent placement; however this approach may not provide durable relief [22-26]. Stent placement which usually helps empty the RHV into the cava can occasionally occlude the MHV or LHV requiring additional smaller stents in these other HVs. With intact liver function, if HV stenting is not an option and multiple venoplasties have failed, then a surgical mesocaval or a side to side splenorenal shunt can be attempted. If the liver function is not preserved, then consider a retransplant with possible transjugular intrahepatic porto-systemic shunt (TIPS) placement for temporary control of the RA. The use of TIPS is associated with an increased risk of infection as well as the development of liver failure $[27,28]$. Renal function and bilirubin should be checked carefully prior to selecting a patient for TIPS. The TIPS may need to be placed just into the cava to allow for decompression of the PHT caused at least partially by the venous outflow obstruction (Figure 6).

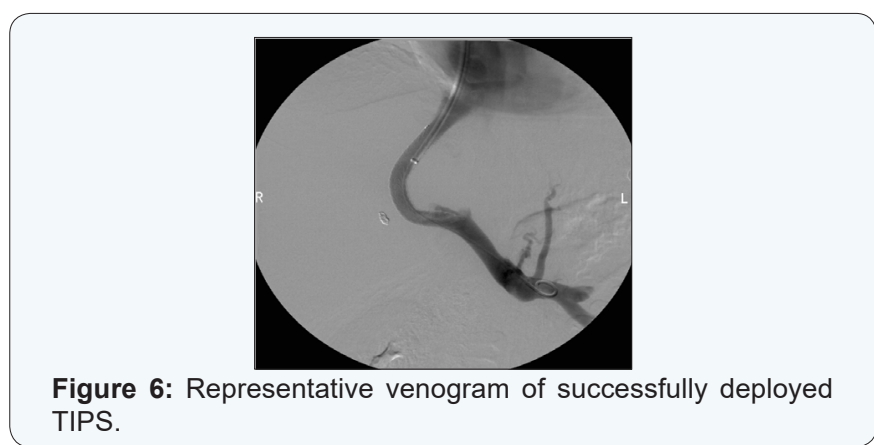

LeVeen shunt is one of the options used in treating RA, however, risks include infection, disseminated intravascular coagulopathy, thrombosis and heart failure [29].

\section{Conclusion}

The description of our approach to refractory ascites after liver transplantation is based on small series and case reports in the literature as well as our clinical observations over several decades of dealing with this uncommon complex post-transplant complication. The proposed algorithm first separates the origin of the RA into cirrhotic and non-cirrhotic etiologies. Once the practitioner has determined a non-cirrhotic etiology for posttransplant RA, we offer a stepwise approach to diagnosis and management focused on the vascular complications commonly encountered.

\section{Disclosure}

The authors of the manuscript have no conflict of interest to disclose as described by the American Journal of Transplantation.

\section{References}

1. Moore KP, Wong F, Gines P, Bernardi M, Ochs A, et al. (2003) The management of ascites in cirrhosis: report on the consensus conference of the International Ascites Club. Hepatology 38(1): 258-266.

2. Thuluvath PJ, Bal JS, Mitchell S, Lund G, Venbrux A (2003) TIPS for management of refractory ascites: response and survival are both unpredictable. Dig Dis Sci 48(3): 542-550.

3. Suzuki H, Stanley AJ (2001) Current management and novel therapeutic strategies for refractory ascites and hepatorenal syndrome. QJM 94(6): 293-300.

4. Chutaputti A (2002) Management of refractory ascites and hepatorenal syndrome. J Gastroenterol Hepatol 17(4): 456-461.

5. Stewart CA, Wertheim J, Olthoff K, Furth EE, Brensinger C, et al. (2004) Ascites after liver transplantation--a mystery. Liver Transpl 10(5): 654660.

6. Abouljoud M, Yoshida A, Kim D, Jerius J, Arenas J, et al. (2005) Transjugular intrahepatic portosystemic shunts for refractory ascites after liver transplantation. Transplant Proc 37(2): 1248-1250.

7. Nishida S, Gaynor JJ, Nakamura N, Butt F, Illanes HG, et al. (2006) Refractory ascites after liver transplantation: an analysis of 1058 liver transplant patients at a single center. Am J Transplant 6(1): 140-149.

8. Noble-Jamieson G, Jamieson N, Barnes ND (1991) Ultrafiltration for intractable ascites after liver transplantation. Arch Dis Child 66(8): 988-989. 
9. Navasa M, Feu F, García-Pagán JC, Jiménez W, Llach J, et al. (1993) Hemodynamic and humoral changes after liver transplantation in patients with cirrhosis. Hepatology 17(3): 355-360.

10. Henderson JM, Gilmore GT, Mackay GJ, Galloway JR, Dodson TF, et al. (1992) Hemodynamics during liver transplantation: the interactions between cardiac output and portal venous and hepatic arterial flows. Hepatology 16(3): 715-718.

11. Paulsen AW, Klintmalm GB (1992) Direct measurement of hepatic blood flow in native and transplanted organs, with accompanying systemic hemodynamics. Hepatology 16(1): 100-111.

12. Henderson JM (1993) Abnormal splanchnic and systemic hemodynamics of end-stage liver disease: what happens after liver transplantation? Hepatology 17(3): 514-516.

13. Vallance P, Moncada S (1991) Hyperdynamic circulation in cirrhosis: a role for nitric oxide? Lancet 337(8744): 776-778.

14. Parrilla P, Sánchez-Bueno F, Figueras J, Jaurrieta E, Mir J, et al. (1999) Analysis of the complications of the piggy-back technique in 1,112 liver transplants. Transplantation 67(9): 1214-1217.

15. Cirera I, Navasa M, Rimola A, García-Pagán JC, Grande L, et al. (2000) Ascites after liver transplantation. Liver Transpl 6(2): 157-162.

16. Gotthardt DN, Weiss KH, Rathenberg V, Schemmer P, Stremmel W, et al. (2013) Persistent ascites after liver transplantation: etiology, treatment and impact on survival. Ann Transplant 18: 378-383.

17. Kok T, Peeters PM, Hew JM, Martijn A, Koetse HA, et al. (1995) Doppler ultrasound and angiography of the vasculature of the liver in children after orthotopic liver transplantation: a prospective study. Pediatr Radiol 25(7): 517-524.

18. Cheng YF, Huang TL, Chen CL, Lee TY, Chen TY, et al. (1998) Intraoperative Doppler ultrasound in liver transplantation. Clin Transplant12(4): 292-299.

19. Park KB, Choo SW, Do YS, Shin SW, Cho SG, et al. (2005) Percutaneous angioplasty of portal vein stenosis that complicates liver transplantation: the mid-term therapeutic results. Korean J Radiol 6(3): 161-166.
20. Shibata T, Itoh K, Kubo T, Maetani Y, Togashi K, et al. (2005) Percutaneous transhepatic balloon dilation of portal venous stenosis in patients with living donor liver transplantation. Radiology 235(3): 1078-1083.

21. Funaki B, Rosenblum JD, Leef JA, Zaleski GX, Farrell T, et al. (2000) Percutaneous treatment of portal venous stenosis in children and adolescents with segmental hepatic transplants: long-term results. Radiology 215(1): 147-151.

22. Sze DY, Semba CP, Razavi MK, Kee ST, Dake MD (1999) Endovascular treatment of hepatic venous outflow obstruction after piggyback technique liver transplantation. Transplantation 68(3): 446-449.

23. Ko GY, Sung KB, Yoon HK, Kim JH, Song HY, et al. (2002) Endovascular treatment of hepatic venous outflow obstruction after living-donor liver transplantation. J Vasc Interv Radiol 13(6): 591-599.

24. Rerksuppaphol S, Hardikar W, Smith AL, Wilkinson JL, Goh TH, et al. (2004) Successful stenting for Budd-Chiari syndrome after pediatric liver transplantation: a case series and review of the literature. Pediatr Surg Int 20(2): 87-90.

25. Nazarian GK, Austin WR, Wegryn SA, Bjarnason H, Stackhouse DJ, et al. (1996) Venous recanalization by metallic stents after failure of balloon angioplasty or surgery: four-year experience. Cardiovasc Intervent Radiol 19(4): 227-233.

26. Wang SL, Sze DY, Busque S, Razavi MK, Kee ST, et al. (2005) Treatment of hepatic venous outflow obstruction after piggyback liver transplantation. Radiology 236(1): 352-359.

27. Urbani L, Catalano G, Cioni R, Petruzzi P, Bindi L, et al. (2003) Management of massive and persistent ascites and/or hydrothorax after liver transplantation. Transplant Proc 35(4): 1473-1475.

28. Lerut JP, Goffette P, Molle G, Roggen FM, Puttemans T, et al. (1999) Transjugular intrahepatic portosystemic shunt after adult liver transplantation: experience in eight patients. Transplantation 68(3): 379-384.

29. Mabrut JY, de la Roche E, Adham M, Ducerf C, et al. (1998) Peritoneovenous diversion using the LeVeen shunt in the treatment of refractory ascites after liver transplantation. Ann Chir 52(7): 612-617. 